PROCEEDINGS OF THE

AMERICAN MATHEMATICAL SOCIETY

Volume 127, Number 8, Pages 2417-2422

S 0002-9939(99)04851-0

Article electronically published on April 9, 1999

\title{
EXACT ESTIMATES FOR INTEGRALS INVOLVING DIRICHLET SERIES WITH NONNEGATIVE COEFFICIENTS
}

\author{
FERENC MÓRICZ
}

(Communicated by Frederick W. Gehring)

Abstract. We consider the Dirichlet series

$$
\sum_{k=2}^{\infty} a_{k} k^{-1-x}=: f(x), \quad x>0,
$$

with coefficients $a_{k} \geq 0$ for all $k$. Among others, we prove exact estimates of certain weighted $L^{p}$-norms of $f$ on the unit interval $(0,1)$ for any $0<p<\infty$, in terms of the coefficients $a_{k}$. Our estimation is based on the close relationship between Dirichlet series and power series. This enables us to derive exact estimates for integrals involving the former one by relying on exact estimates for integrals involving the latter one. As a by-product, we obtain an analogue of the Cauchy-Hadamard criterion of (absolute) convergence of the more general Dirichlet series

with complex coefficients $c_{k}$.

$$
\sum_{k=1}^{\infty} c_{k} k^{-z}, \quad z:=x+i y
$$

\section{INTRODUCTION}

We shall consider the Dirichlet series with nonnegative coefficients

$$
\sum_{k=2}^{\infty} a_{k} k^{-1-x}=: f(x), \quad a_{k} \geq 0,
$$

assuming that the series on the left-hand side converges for all $x>0$. In Section 3 , we shall give a necessary and sufficient condition to ensure this convergence (see (3.9) there).

There is a definite interest in the literature (see, for example, [1], [2], [4], and [6]) to estimate certain weighted integrals of $\phi(f(x))$ over the unit interval $(0,1)$, or over the half real axis $(0, \infty)$, where $\phi$ and the weight functions in question are specified as follows.

Given $p \geq q>0$, we shall write $\phi \in \Delta(p, q)$ if $\phi(t)$ is a nonnegative function defined on $[0, \infty), \phi(0)=0, \phi(t) t^{-p}$ is nonincreasing, and $\phi(t) t^{-q}$ is nondecreasing

Received by the editors November 19, 1997.

1991 Mathematics Subject Classification. Primary 30B50; Secondary 30 B10.

Key words and phrases. Dirichlet series, power series, Cauchy-Hadamard criterion for Dirichlet series, line of convergence, $L^{p}$-behavior, weight function, slowly decreasing function, Cauchy condensation principle.

This research was partially supported by the Hungarian National Foundation for Scientific Research under Grant T 016393.

(C)1999 American Mathematical Society 
on $(0, \infty)$. We write $\phi \in \Delta$ if $\phi \in \Delta(p, q)$ for some $p \geq q>0$, but the values of $p$ and $q$ are unimportant in the question concerned.

Clearly, if $\phi \in \Delta$, then $\phi(t)$ is nondecreasing on $[0, \infty)$. Even more is true: If $\phi \in \Delta(p, q)$ for some $p \geq q>0$, then

$$
v^{p} \phi(t) \leq \phi(v t) \leq v^{q} \phi(t) \quad \text { for } \quad 0 \leq v \leq 1 \quad \text { and } \quad t \geq 0 .
$$

The weight functions in this paper are "slowly decreasing." More precisely, we shall write $\lambda \in \Lambda$ if $\lambda(t)$ is a positive, nonincreasing function on $[1, \infty)$ and such that

$$
0<\liminf _{t \rightarrow \infty} \frac{\lambda(2 t)}{\lambda(t)} \leq \limsup _{t \rightarrow \infty} \frac{\lambda(2 t)}{\lambda(t)}<1 .
$$

\section{NeW RESULtS}

We shall use the following notations:

$$
\begin{aligned}
I_{n} & :=\left\{2^{n}, 2^{n}+1, \ldots, 2^{n+1}-1\right\}, \quad n=1,2, \ldots ; \\
\alpha_{n} & :=2^{-n} \sum_{k \in I_{n}} a_{k}, \quad A_{n}:=\sum_{m=1}^{n} \alpha_{m}, \quad n=1,2, \ldots ;
\end{aligned}
$$

where $\left\{a_{k}: k=2,3, \ldots\right\}$ is the sequence of coefficients in (1.1).

Our main result is the following

Theorem 1. If $\lambda \in \Lambda, \Phi \in \Delta$, and $f(x)$ is defined in (1.1), then there exists a positive constant $K$ depending only on $\phi$ and $\lambda$ such that

$$
\begin{aligned}
K^{-1} & \sum_{n=0}^{\infty} \lambda\left(2^{n}\right) \phi\left(A_{2^{n+1}-1}\right) \\
& \leq \int_{0}^{1} \lambda\left(\left(1-2^{-x}\right)^{-1}\right)\left(1-2^{-x}\right)^{-1} \phi(f(x)) d x \\
& \leq K \sum_{n=0}^{\infty} \lambda\left(2^{n}\right) \phi\left(\sum_{k \in I_{n}} \alpha_{k}\right) .
\end{aligned}
$$

It is plain that estimate (2.3) remains valid if $A_{2^{n+1}-1}$ is replaced by $\sum_{k \in I_{n}} \alpha_{k}$ on the left-most side and/or if $\sum_{k \in I_{n}} \alpha_{k}$ is replaced by $A_{2^{n+1}-1}$ on the right-most side of (2.3).

First, we consider the special case, where

$$
\lambda(t):=t^{-\gamma}, \quad \gamma>0 .
$$

Making use of the Cauchy condensation principle yields the following

Corollary 1. If $\gamma>0$ and $\phi \in \Delta$, then there exists a positive constant $K$ depending only on $\gamma$ and $\phi$ such that

$$
K^{-1} \sum_{n=1}^{\infty} \frac{\phi\left(A_{n}\right)}{n^{\gamma+1}} \leq \int_{0}^{1}\left(1-2^{-x}\right)^{\gamma-1} \phi(f(x)) d x \leq K \sum_{n=1}^{\infty} \frac{\phi\left(A_{n}\right)}{n^{\gamma+1}} .
$$

Since

$$
\frac{x}{2} \ln 2 \leq 1-2^{-x} \leq x \ln 2 \quad \text { for } \quad 0 \leq x \leq 1,
$$


from (2.4) it follows immediately that

$$
x^{\gamma-1} \phi(f(x)) \in L^{1}(0,1) \text { if and only if } \sum_{n=1}^{\infty} \frac{\phi\left(A_{n}\right)}{n^{\gamma+1}}<\infty .
$$

Second, we consider the even more special case, where $\gamma:=1$, i.e., let $\lambda(t):=t^{-1}$.

Corollary 2. If $\phi \in \Delta$, then there exists a positive constant $K$ depending only on $\phi$ such that

$$
K^{-1} \sum_{n=1}^{\infty} \frac{\phi\left(A_{n}\right)}{n^{2}} \leq \int_{0}^{1} \phi(f(x)) d x \leq \int_{0}^{\infty} \phi(f(x)) d x \leq K \sum_{n=1}^{\infty} \frac{\phi\left(A_{n}\right)}{n^{2}}
$$

In particular, if $\phi(t):=t^{p}$ for some $p>0$, then (2.5) provides an exact estimate for the $L^{p}$-norm of the sum of the Dirichlet series (1.1).

\section{Auxiliary Results}

Our estimation is based on the close relationship between Dirichlet series and power series, which enables us to derive exact estimates for integrals involving the former one by making use of exact estimates for integrals involving the latter one, which has been available in the literature.

To go into details, consider the power series with nonnegative coefficients

$$
\sum_{k=1}^{\infty} b_{k} x^{k}=: g(x), \quad b_{k} \geq 0
$$

assuming that the series on the left-hand side converges for all $0 \leq x<1$. According to the Cauchy-Hadamard criterion, the condition

$$
\limsup _{k \rightarrow \infty}\left|b_{k}\right|^{1 / k} \leq 1
$$

is necessary and sufficient in order that the radius of convergence of series (3.1) be at least 1 .

We shall use the following notation:

$$
\beta_{n}:=\sum_{k \in I_{n}} b_{k}, \quad s_{n}:=\sum_{k=1}^{n} b_{k},
$$

where $I_{n}$ is defined in $(2.1)$.

The next theorem was proved by Leindler [3].

Theorem 2. If $\lambda \in \Lambda, \phi \in \Delta$, and $g(x)$ is defined in (3.1), then there exists a positive constant $K$ depending only on $\lambda$ and $\phi$ such that

$$
\begin{aligned}
K^{-1} & \sum_{n=0}^{\infty} \lambda\left(2^{n}\right) \phi\left(s_{2^{n+1}-1}\right) \\
& \leq \int_{0}^{1} \lambda\left((1-x)^{-1}\right)(1-x)^{-1} \phi(g(x)) d x \\
& \leq K \sum_{n=0}^{\infty} \lambda\left(2^{n}\right) \phi\left(\beta_{n}\right) .
\end{aligned}
$$


The first inequality in (3.4) was first proved by Mateljevic and Pavlovic [5] in the special case $\lambda(t):=t^{-\gamma}$ for some $\gamma>0$. The second inequality was proved also by them with the seemingly greater quantity $\phi\left(s_{2^{n+1}-1}\right)$ instead of $\phi\left(\beta_{n}\right)$. However, it is easy to see that, under the conditions of Theorem 2, the sums of the series

$$
\sum_{n=0}^{\infty} \lambda\left(2^{n}\right) \phi\left(s_{2^{n+1}-1}\right) \quad \text { and } \quad \sum_{n=0}^{\infty} \lambda\left(2^{n}\right) \phi\left(\beta_{n}\right)
$$

are of the same order of magnitude.

Analyzing the proofs in [3] and [5], one can conclude the following improvement of the first inequality in (3.4).

Lemma 1. Let

$$
r_{n}:=1-2^{-n}, \quad n=0,1,2, \ldots .
$$

Under the conditions of Theorem 2, we have

$$
\begin{aligned}
K^{-1} & \sum_{n=n_{0}}^{\infty} \lambda\left(2^{n}\right) \phi\left(s_{2^{n+1}-1}\right) \\
& \leq \int_{r_{n_{0}}}^{1} \lambda\left((1-x)^{-1}\right)(1-x)^{-1} \phi(g(x)) d x, \quad n_{0}=0,1,2, \ldots ;
\end{aligned}
$$

with the same constant $K$ as in (3.4).

The next auxiliary result is of crucial importance for our purposes, but it is of special interest in itself. It makes it possible to pass from power series into Dirichlet series.

Lemma 2. Given a Dirichlet series (1.1), introduce the power series

$$
\sum_{n=1}^{\infty} \alpha_{n} u^{n}=: h(u),
$$

where $\alpha_{n}$ is defined in (2.2). If the radius of convergence of the series on the left-hand side in (3.6) is at least 1 , then

$$
2^{-1-x} h\left(2^{-x}\right) \leq f(x) \leq h\left(2^{-x}\right) \quad \text { for } \quad x>0 .
$$

Proof. By (1.1), (2.1), and (2.2), it is plain that

$$
\begin{aligned}
f(x) & =\sum_{n=1}^{\infty} \sum_{k \in I_{n}} a_{k} k^{-1-x} \\
& \leq \sum_{n=1}^{\infty} 2^{n(-1-x)} 2^{n} \alpha_{n}=h\left(2^{-x}\right) .
\end{aligned}
$$

On the other hand,

$$
\begin{aligned}
f(x) & \geq \sum_{n=1}^{\infty} 2^{(n+1)(-1-x)} 2^{n} \alpha_{n} \\
& =2^{-1} \sum_{n=1}^{\infty} \alpha_{n} 2^{-(n+1) x}=2^{-1-x} h\left(2^{-x}\right) .
\end{aligned}
$$


Combining Lemma 2 with the Cauchy-Hadamard criterion, while making use of the obvious inequality

$$
\frac{1}{2} \alpha_{n} \leq \sum_{k \in I_{n}} \frac{a_{k}}{k} \leq \alpha_{n}
$$

yields the following

Lemma 3. If

$$
2^{x}>\limsup _{n \rightarrow \infty}\left\{\sum_{k \in I_{n}} \frac{a_{k}}{k}\right\}^{1 / n},
$$

then the Dirichlet series (1.1) converges. If the inequality sign is reversed in (3.8), then series (1.1) diverges.

In particular, the Dirichlet series (1.1) converges for all $x>0$ if and only if

$$
\limsup _{n \rightarrow \infty}\left\{\sum_{k \in I_{n}} \frac{a_{k}}{k}\right\}^{1 / n} \leq 1 .
$$

As a by-product, we obtain an analogue of the Cauchy-Hadamard criterion of (absolute) convergence of the more general Dirichlet series

$$
\sum_{k=2}^{\infty} c_{k} k^{-z}, \quad z:=x+i y
$$

where $\left\{c_{k}\right\}$ is a sequence of complex numbers.

Denote by $\Theta$ the supremum of those $x:=\operatorname{Re} z$, for which series (3.10) converges. By what we have discussed above, it is clear that $\Theta$ is determined by the equation

$$
2^{\Theta-1}=\limsup _{n \rightarrow \infty}\left\{\sum_{k \in I_{n}} \frac{\left|c_{k}\right|}{k}\right\}^{1 / n} .
$$

(In $(1.1)$ we have $z:=1+x$.) Furthermore, the line given by the equation $z=\Theta$ can be viewed as the "line of absolute convergence," which means that series (3.10) converges absolutely for all $z$ with $x:=\operatorname{Re} z>\Theta$, while it diverges for all $z:=x<$ $\Theta$.

\section{Proofs of Theorem 1 and Corollaries 1 and 2}

Proof of Theorem 1. (i) By Lemma 2, Theorem 2, and (2.2), while introducing $u:=2^{-x}$, we have

$$
\begin{aligned}
I: & =\int_{0}^{1} \lambda\left(\left(1-2^{-x}\right)^{-1}\right)\left(1-2^{-x}\right)^{-1} \phi(f(x)) d x \\
& \leq \int_{1 / 2}^{1} \lambda\left((1-u)^{-1}\right)(1-u)^{-1} \phi(h(u)) \frac{d u}{u \ln 2} \\
& \leq \frac{2 K}{\ln 2} \sum_{n=0}^{\infty} \lambda\left(2^{n}\right) \phi\left(\sum_{k \in I_{n}} \alpha_{k}\right) .
\end{aligned}
$$

This proves the second inequality in (2.3). 
(ii) By Lemmas 1 and 2, (1.2), and (2.2), we get similarly that

$$
\begin{aligned}
I & \geq \int_{1 / 2}^{1} \lambda\left((1-u)^{-1}\right)(1-u)^{-1} \phi\left(2^{-1} u h(u)\right) \frac{d u}{u \ln 2} \\
& \geq \frac{K^{-1}}{2^{p} \ln 2} \min \left\{1, \frac{1}{2^{p-1}}\right\} \sum_{n=1}^{\infty} \lambda\left(2^{n}\right) \phi\left(A_{2^{n+1}-1}\right) .
\end{aligned}
$$

Hence the first inequality in (2.3) follows easily.

Proof of Corollary 1. From (2.3) it follows immediately that

$$
\begin{aligned}
K^{-1} \sum_{n=0}^{\infty} 2^{-n \gamma} \phi\left(A_{2^{n+1}-1}\right) & \leq \int_{0}^{1}\left(1-2^{-x}\right)^{\gamma-1} \phi(f(x)) d x \\
& \leq K \sum_{n=0}^{\infty} 2^{-n \gamma} \phi\left(A_{2^{n+1}-1}\right) .
\end{aligned}
$$

Making use of the Cauchy condensation principle yields

$$
\sum_{n=0}^{\infty} 2^{-n \gamma} \phi\left(A_{2^{n+1}-1}\right) \geq \sum_{n=0}^{\infty} \sum_{k \in I_{n}} \frac{\phi\left(A_{k}\right)}{k^{\gamma+1}}=\sum_{k=1}^{\infty} \frac{\phi\left(A_{k}\right)}{k^{\gamma+1}} .
$$

This proves the first inequality in (2.4).

The second inequality in (2.4) can be proved analogously.

Proof of Corollary 2. It follows immediately from Corollary 1 when $\gamma=1$, except for the right-most inequality in (2.5). To prove it, let us observe that

$$
f(x+j) \leq 2^{-j} f(x) \quad \text { for } \quad x>0 \text { and } j=1,2, \ldots .
$$

Thus, by (1.2), we have

$$
\int_{0}^{\infty} \phi(f(x)) d x \leq \sum_{j=0}^{\infty} \int_{0}^{1} \phi\left(2^{-j} f(x)\right) d x \leq\left(1-2^{-q}\right)^{-1} \int_{0}^{1} \phi(f(x)) d x .
$$

This proves the right-most inequality in (2.5).

\section{REFERENCES}

1. L. Leindler, Generalizations of some theorems of Mulholland concerning Dirichlet series, Acta Sci. Math. (Szeged) 57(1993), 401-418. MR 94j:41010

2. L. Leindler, Improvements of some theorems of Mulholland concerning Dirichlet series, Acta Sci. Math. (Szeged), 58(1993), 281-297. MR 95g:40002

3. L. Leindler, On power series with positive coefficients, Analysis Math. 20 (1994), 205-211. MR 95k:40010

4. L. Leindler and A. Meir, Inequalities concerning Dirichlet series and integrals, Acta Sci. Math. (Szeged) 59(1994), 209-220. MR 95h:26025

5. M. Mateljevic and M. Pavlovic, $L^{p}$-behavior of power series with positive coefficients and Hardy spaces, Proc. Amer. Math. Soc. 87 (1983), 309-316. MR 84g:30034

6. H. P. Mulholland, Some theorems on Dirichlet series with positive coefficients and related integrals, Proc. London Math. Soc. 29(1929), 281-292.

Bolyai Institute, University of Szeged, Aradi Vertanuk Tere 1, 6720 Szeged, Hungary

E-mail address: moricz@math.u-szeged.hu 